\title{
The 14-3-3 (YWHA) Proteins in Signalling and Development of the Fruit Fly, Drosophila melanogaster
}

\author{
Santanu De \\ Department of Biological Sciences, Halmos College of Natural Sciences and Oceanography, \\ Nova Southeastern University
}

* Corresponding author email: sde@nova.edu

Received: 07 November 2019 / Revised: 01 December 2019 / Accepted: 07 December 2019 / Published: 08 December 2019

\begin{abstract}
The 14-3-3 (YWHA or Tyrosine 3-Monooxygenase/Tryptophan 5-Monooxygenase Activation proteins) are a family of highly conserved, homologous proteins critical to diverse cellular events including cell cycle, signal transduction and embryonic development. Various species-specific isoforms of 14-3-3 exist, encoded by separate genes. They are expressed in a wide variety of organisms ranging from plants to animals, including the fruit fly or Drosophila melanogaster. Drosophila is one of the most universally accepted model systems to study complex cellular mechanisms of signalling and development. However, regulation of these processes in fruit flies by the 14-3-3 proteins have not been entirely understood. This mini review encapsulates the expression, distribution, interactions and regulatory roles of the 14-3-3 proteins in Drosophila. The analysis would help to elucidate some of the molecular bases of key cell-signalling mechanisms and development.
\end{abstract}

Keywords: 14-3-3, YHWA, Drosophila, signalling, development.

\section{Introduction}

The 14-3-3 proteins (YWHA or Tyrosine 3Monooxygenase/Tryptophan 5-Monooxygenase Activation proteins) are a conserved, homologous family of acidic proteins expressed abundantly and ubiquitously in diverse eukaryotic cells and organisms ranging from plants to mammals [1]. They were first identified during a systematic classification of brain proteins. The name '14-33' was used to denote the elution fraction comprising these proteins after DEAE-cellulose chromatography and their migration position after subsequent starch gel electrophoresis. These proteins are known regulators in important physiological and cellular events including metabolism, transcription, signal transduction, cell cycle control, apoptosis, protein trafficking, stress responses, malignant transformation and embryonic development. Over 200 proteins that may interact with 14-3-3 have been detected by proteomic and biochemical methods [2-5].
Protein 14-3-3 exists mainly as homo- or heterodimers with a monomeric molecular mass of approximately $30 \mathrm{kDa}$ [2]. Different 14-3-3 isoforms can interact with the same ligand and so are somewhat interchangeable; however, although isoforms of 14-3-3 often bind the same protein, there are some indications that homodimers of different types or even heterodimers of 14-3-3 may have different roles in the regulation or sequestering of proteins [6,7]. Dimerization of $14-3-3$ s is important, because point mutations that disrupt 14-3-3 dimers can impair the regulatory functions of 14-3-3s [8, 9].

The 14-3-3 proteins bind to a multitude of signalling molecules by phosphorylationdependent mechanisms. They complement or supplement intracellular events involving phosphorylation-dependent switching or proteinprotein interaction $[4,10]$. The 14-3-3s interact with proteins containing phosphoserine and phospho-threonine residues with RSXpSXP and 
$\mathrm{X}(\mathrm{Y} / \mathrm{F}) \mathrm{XpSXP}$ motifs $[11,12]$. Most of the binding partners of 14-3-3 are phosphorylated, however, phosphorylation-dependent sites that differ significantly from these motifs have been reported, and some interactions of 14-3-3 do exist independent of phosphorylation [6]. The effect of 14-3-3 binding has diverse cellular effect(s) depending on the nature of the ligand. Changes in phosphorylation status of the 14-3-3 binding motif on target proteins may result in changes in binding patterns. For instance, dephosphorylation of certain serine residues on target proteins may release the interaction with 143-3; likewise, serine phosphorylation on certain target proteins may lead to 14-3-3-target interactions. Proteomic analysis of interphase and mitotic HeLa cells detected a plethora of 14-3-3binding proteins involved in cell cycle regulation, signalling, metabolism, protein synthesis, nucleic acid binding, chromatin structure, protein folding, proteolysis, nucleolar function, and nuclear transport as well as several other cellular processes [5].

The fruit fly or Drosophila is an easily available, cost effective model organism with rapid generation time and offering excellent genetic tools. It is one of the most commonly used model organisms to study signalling and development $[13,14]$. On the other hand, the 14-3-3 proteins are known to control mitosis in mammalian cells and meiosis in amphibians. Recent works place 14-3-3s as critical molecular controllers in Drosophila [14-19]. However, as a major challenge to animal physiological studies, the significance of the 14-33 proteins in fruit fly development and signalling has not been entirely reviewed. Therefore, this paper aims at presenting a comprehensive minireview of relevant research findings focused on the expression, distribution, binding partners and functional roles of the 14-3-3 proteins in the regulation of molecular mechanisms associated with cellular signalling and development in Drosophila.

\section{Evolution of 14-3-3 proteins in Drosophila}

The evolutionary history of $14-3-3$ protein apparently is characterized by a distinct divergence of animal and plant isoforms from a common ancestral sequence [20]. Large domains of the protein isoforms remain conserved in plant as well as animal lineages despite their independent evolutionary pathways since the ancestral split. In the course of research investigating proteins co-expressed with the prohormone, proopiomelanocortin in the melanotrope cells of Xenopus intermediate pituitary gland, alignment of a Xenopus 14-3-3 protein encoded by a pituitary complementary DNA (cDNA) with known 14-33 polypeptide of Drosophila, plants and mammals and with a mammalian protein kinase $\mathrm{C}$ indicated marked conservation (60-88\%) of the neuronspecific 14-3-3-related proteins throughout the evolutionary tree of eukaryotes [21].

In mammals, to date, seven isoforms of 14-3-3 have been discovered that are encoded by separate genes, with isoform-specific roles in development across several species of animals [22-31]. The phosphorylated forms of $14-3-3 \beta$ and $14-3-3 \gamma$ were initially described as $14-3-3 \alpha$ and $14-3-3 \delta$, respectively [32]. However, Drosophila contains only two 14-3-3 genes: leonardo, an ortholog of the mammalian 14-3-35 (88\% identity), and D14$3-3 \varepsilon$, an ortholog of 14-3-3e [33]. The two fly genes belong to two different 14-3-3 conservation groups [20, 33].

\section{The 14-3-3 proteins in Drosophila development and signalling}

The 14-3-3 proteins appear to regulate the interaction between translationally controlled tumour protein (Tctp) and Ras homolog enriched in brain (Rheb) GTPase proteins for organ growth in Drosophila [18]. During transcription elongation in Drosophila, histone phosphorylation and acetylation are linked through crosstalk mediated by the 14-3-3 proteins which interact with the acetyltransferase elongator protein 3 (Elp3) [34]. Moreover, it has been shown that in Drosophila, 143-3-binding sites are produced upon phosphorylation of various proteins by partitioning defective proteins (PAR-1), kinases that are known to be necessary for anteriorposterior (A-P) axis determination [35]. Thus, interaction of 14-3-3 protein with its substrates mediates the activity of PAR-1. Complementary cortical domains in polarized cells have been 
reported to be established by Drosophila PAR-1 and 14-3-3 that inhibit Bazooka/PAR-3 [36].

During oogenesis in Drosophila, 14-3-3 has been shown to translate a spatial cue provided by Aurora B kinase to target non-claret disjunctional (Ncd) kinesin-like protein (necessary for female meiotic chromosomal segregation) selectively to the meiotic spindle of oocytes $[15,16]$. This demonstrates a novel mechanism by which chromosomes regulate acentrosomal spindle assembly via a series of phosphorylation events.

Recent genetic and biochemical experiments show that 14-3-3 proteins influence planar cell division by acting together with the neoplastic tumor suppressor proteins, Scribbled (Scrib) and Discs large (Dlg) as well as the spindle pole-associated factor, mushroom body defect (Mud) during planar cell division [19]. This work indicated 143-3s as Dlg-interacting proteins, loss of which leads to disorientation of spindle along with disrupted epithelial architecture due to basal cell delamination and apoptosis.

\section{Isoform-specific expression and roles of 14-3-3 in Drosophila development and signalling}

Alternative splicing of the primary transcript of leonardo has been indicated to produce two nearly identical protein isoforms, LEO I and LEO II [37, 38], despite the existence of some tissue specificity [38]. In contrast, a single protein is encoded by D14-3-3ع [39], which appears to be expressed in all developmental stages and tissues of the fly with little over-abundance in the adult brain [38, 40]. D14-3-3e seems necessary for embryonic hatching; however, loss of D14-3-3e can be functionally compensated by excess of a particular LEO isoform in a cellular context-specific manner; thus, functional differences among Drosophila 14-3-3 proteins as well as between the two isoforms of LEO in vivo likely govern differential dimer affinities toward 14-3-3 targets [41]. Protein 14-3-3e has been noticed to be crucial to cellular metabolism by means of molecular crosstalk with ecdysone receptor (EcR) or through direct interaction with metabolic binding partners [17].

The $14-3-3 \varepsilon$ isoform is essential for germ cell migration in Drosophila [42]. In addition, this isoform of 14-3-3 antagonizes the transcription factor FoxO to control growth, apoptosis and longevity in Drosophila [43]. Besides, differentiation in the eye and embryo of the adult fly have been known to be regulated by Rat Sarcoma/ Mitogen-Activated Protein Kinase (RAS/MAPK) signalling pathways where D14-3$3 \varepsilon$ and leonardo have been found important, as outlined below.

It was reported in 2011 that signalling mediated by the small GTPase, Ras in Drosophila is upregulated by $14-3-3 \varepsilon$ [39]. The authors demonstrated through a loss-of-function mutation that 14-3-3e acts to increase the efficiency of RAS1 signalling. Mutations in the gene encoding a Drosophila 14-3$3 \varepsilon$ were isolated as suppressors of the rough eye phenotype resulting from ectopic expression of Ras1 ${ }^{\mathrm{v} 12}$, an activated allele of Ras1. It appears that the $14-3-3 \varepsilon$ protein is engaged in multiple receptor tyrosine kinase (RTK) pathways. This indicates $14-3-3 \varepsilon$ to be a putative general component of the RAS1 signalling cascade. Two regions of 14-3-3e taking part in RAS1 signalling have been revealed by sequence analysis of three dominant-negative alleles. Further evidence suggests partial redundance of the two members of the Drosophila 14-3-3 protein family, $14-3-3 \varepsilon$ and $14-3-3 \zeta$, for RAS1 signalling in animal viability and generation of photoreceptors. Protein $14-3-3 \varepsilon$ is thought to act parallel to or downstream of Rapidly Accelerated Fibrosarcoma (RAF) kinase, but upstream of nuclear factors in signalling mediated by RAS1. Protein 14-3-3e has been shown to be essential for Rab11-positive vesicle function, thereby helping antimicrobial peptide secretion during an innate immune response [44]. Infection with Gram-positive or Gram-negative bacteria leads to reduced survival in D14-3-3e mutants, suggesting a role for D14-3-3 $\varepsilon$ in innate immunity. On the other hand, D14-3-36 or leonardo has been demonstrated as an essential component of the Raf/Ras signalling pathway, is important for the differentiation of photoreceptors, and acts upstream of Raf and downstream of Ras [37]. It has been observed that binding of Raf to the Drosophila 14-3-3 protein leonardo is required for the activation of Raf and that, overexpressed leonardo needs Ras1 to activate D-Raf [45]. The Drosophila 14-3-3ל protein or leonardo has been 
suggested to play a role in regulating synaptic vesicle dynamics, thereby controlling the properties of synaptic modulation to promote the process of learning [46].

Mutant analyses of 14-3-3 $\varepsilon$ and $14-3-3 \zeta$ suggested that $14-3-3 \varepsilon$ is essential for timing of mitosis in undisturbed post-blastoderm cell cycles and to delay mitosis after irradiation, while $14-3-3 \zeta$ is necessary for normal chromosome separation during syncytial mitoses [47]. The study proposed a model where, in the undisturbed cell cycle, 14-33 proteins serve to prepare a threshold for entry into mitosis by inhibiting Cdk1 activity, to suppress mitosis following radiation damage and to augment accurate exit from mitosis. In undisturbed post-blastoderm cell cycles, the timing of mitosis has been noted to be controlled by $\mathrm{D} 14-3-3 \varepsilon$, and in order to delay mitosis upon

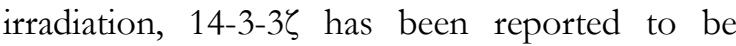
essential during syncytial mitoses for normal chromosome segregation.

\section{Discussion}

The 14-3-3 proteins are highly conserved in evolution across a wide range of species, and exhibit species-specific, developmental stagespecific and tissue-specific similarities and variations in function. Recent studies show 14-3$3 \mathrm{~s}$ as critical molecular regulators in Drosophila [1419]. Specific isoforms of these proteins are expressed and distributed differentially as putative molecular controllers in particular types of cells and organs in a gamut of other species as well such as bull, hamster, Xenopus, horseshoe crab, monkey, rat, turkey and mouse $[28,31,48]$. Proteomic studies of bovine sperm 14-3-3-interactors have identified proteins involved in signalling and metabolism [49]. Furthermore, affinity purification experiments have revealed many of the binding partners of 14-3-3 as key players in cell signalling, transcription, translation, splicing, metabolism and protein trafficking [50].

The broad repertoire of the 14-3-3 interactome suggests the centrality of this class of proteins as a general biochemical regulator. These are key controlling or adapter proteins that may alter the activity of their binding partners, change the associations or interactions of the bound proteins with other proteins, protect protein phosphorylation, promote protein stability and/or alter the intracellular localization or destination of the bound protein. Thus, the 14-3-3 proteins could play a crucial role in coordinating molecular crosstalk among numerous interlinked pathways of cell signalling and development.

\section{Conclusion}

The paper, for the first time, provides a comprehensive short review of research studies analysing the 14-3-3 (YWHA) family of proteins underlying the complex mechanisms of cellular signalling and development in the fruit fly or Drosophila. It is one of the most universally accepted model systems to examine cellular signalling and development at the molecular level; however, regulation of such processes in fruit flies by the 14-3-3 proteins have not been completely elucidated. As a limitation of the study, not much is yet known about the effects of homo- and hetero-dimerization of 14-3-3 isoforms on development or signalling in Drosophila. Further work is recommended to address this question, especially since in several other species the 14-3-3 isoforms have been noted to be interchangeable by interacting with the same ligand. Reports of the isoform-specific, developmental stage-specific and tissue-specific expression, distribution, interactions and functional roles of the 14-3-3 proteins could help unravel the molecular bases of novel cell cycle-regulatory pathways in the fruit fly. Owing to the remarkable evolutionary conserved nature and ubiquitous expression of these homologous proteins across species, implementation of the knowledge about these proteins in Drosopbila could form a foundation for future investigation towards a better understanding of the importance of 14-3-3 in regulating development and signalling across a wide range of organisms, including humans.

\section{Competing Interests}

The author declares no conflict of interest including any financial, personal or other relationships with other people or organizations that could inappropriately influence or be perceived to influence the present work. 
Santanu De, Int. Ann. Sci.; Vol. 9, Issue 1, pp: 80-85, 2020

\section{How to Cite this Article:}

S. De, "The 14-3-3 (YWHA) Proteins in Signalling and Development of the Fruit Fly, Drosophila melanogaster", Int. Ann. Sci., vol. 9, no. 1, pp. 80-85, Dec. 2019. doi:10.21467/ias.9.1.80-85

\section{References}

[1] M. B. Yaffe, "How do 14-3-3 proteins work?-Gatekeeper phosphorylation and the molecular anvil hypothesis," FEBS Lett, vol. 513, no. 1, pp. 53-7, Feb 20 2002.

[2] A. Aitken, "14-3-3 proteins: a historic overview," Semin Cancer Biol, vol. 16, no. 3, pp. 162-72, Jun 2006.

[3] M. Conti et al., "Role of cyclic nucleotide signaling in oocyte maturation," Mol Cell Endocrinol, vol. 187, no. 12, pp. 153-9, Feb 222002.

[4] C. Mackintosh, "Dynamic interactions between 14-3-3 proteins and phosphoproteins regulate diverse cellular processes," Biochem J, vol. 381, no. Pt 2, pp. 329-42, Jul 152004.

[5] S. E. Meek, W. S. Lane, and H. Piwnica-Worms, "Comprehensive proteomic analysis of interphase and mitotic 14-3-3-binding proteins," J Biol Chem, vol. 279, no. 31, pp. 32046-54, Jul 302004.

[6] A. Aitken, "Functional specificity in 14-3-3 isoform interactions through dimer formation and phosphorylation. Chromosome location of mammalian isoforms and variants," Plant Mol Biol, vol. 50, no. 6, pp. 993-1010, Dec 2002

[7] A. Benzinger, N. Muster, H. B. Koch, J. R. Yates, 3rd, and H. Hermeking, "Targeted proteomic analysis of 14-3-3 sigma, a p53 effector commonly silenced in cancer," Mol Cell Proteomics, vol. 4, no. 6, pp. 785-95, Jun 2005.

[8] C. M. Cahill et al., "Phosphatidylinositol 3-kinase signaling inhibits DAF-16 DNA binding and function via 14-3-3-dependent and 14-3-3-independent pathways," $J$ Biol Chem, vol. 276, no. 16, pp. 13402-10, Apr 202001.

[9] G. Tzivion, Z. Luo, and J. Avruch, "A dimeric 14-3-3 protein is an essential cofactor for Raf kinase activity," Nature, vol. 394, no. 6688, pp. 88-92, 1998/07/01 1998.

[10] M. K. Dougherty and D. K. Morrison, "Unlocking the code of 14-3-3," J Cell Sci, vol. 117, no. Pt 10, pp. 1875 84, Apr 152004

[11] K. Rittinger et al., "Structural analysis of 14-3-3 phosphopeptide complexes identifies a dual role for the nuclear export signal of 14-3-3 in ligand binding," Mol Cell, vol. 4, no. 2, pp. 153-66, Aug 1999.

[12] M. B. Yaffe et al., "The structural basis for 14-33:phosphopeptide binding specificity," Cell, vol. 91, no. 7, pp. 961-71, Dec 261997.

[13] A. Singh and K. D. Irvine, "Drosophila as a model for understanding development and disease," Dev Dyn, vol. 241, no. 1, pp. 1-2, Jan 2012.

[14] S. Ahmed-de-Prado and A. Baonza, "Drosophila as a Model System to Study Cell Signaling in Organ Regeneration," Biomed Res Int, vol. 2018, p. 7359267, 2018.

[15] M. Dasso, "Catch and release: 14-3-3 controls Ncd in meiotic spindles," J Cell Biol, vol. 216, no. 10, pp. 30033005 , Oct 22017.

[16] R. Beaven et al., "14-3-3 regulation of Ncd reveals a new mechanism for targeting proteins to the spindle in oocytes," J Cell Biol, vol. 216, no. 10, pp. 3029-3039, Oct 22017.
[17] Y. S. Ng et al., "Proteome Analysis of Drosophila Mutants Identifies a Regulatory Role for 14-3-3epsilon in Metabolic Pathways," J Proteome Res, vol. 16, no. 5, pp. 1976-1987, May 52017.

[18] T. P. Le, L. T. Vuong, A.-R. Kim, Y.-C. Hsu, and K.-W. Choi, "14-3-3 proteins regulate Tctp-Rheb interaction for organ growth in Drosophila," Nat Commun., vol. 7, no. 1, p. 11501, 2016/05/06 2016.

[19] Y. I. Nakajima, Z. T. Lee, S. A. McKinney, S. K. Swanson, L. Florens, and M. C. Gibson, "Junctional tumor suppressors interact with 14-3-3 proteins to control planar spindle alignment," J Cell Biol, vol. 218, no. 6, pp. 18241838, Jun 32019.

[20] W. Wang and D. C. Shakes, "Molecular evolution of the 14-3-3 protein family," J Mol Evol, vol. 43, no. 4, pp. 38498, Oct 1996

[21] G. J. Martens, P. A. Piosik, and E. H. Danen, "Evolutionary conservation of the 14-3-3 protein," Biochem Biophys Res Commun, vol. 184, no. 3, pp. 14569, May 151992.

[22] T. Ichimura et al., "Molecular cloning of cDNA coding for brain-specific 14-3-3 protein, a protein kinasedependent activator of tyrosine and tryptophan hydroxylases," Proc Natl Acad Sci U S A, vol. 85, no. 19, pp. 7084-8, Oct 1988.

[23] S. De, "Protein 14-3-3 (YWHA) isoforms and their roles in regulating mouse oocyte maturation," Kent State University, 2014.

[24] S. De, S. Davis, D. Letwin, C. Mozena, and D. Kline, "Protein 14-3-3 eta (YWHAH) is essential for normal meiotic spindle assembly during in vitro maturation of mouse oocytes," Mol Biol Cell, vol. 23, 2012.

[25] S. De and D. Kline, "Evidence for the requirement of 143-3eta (YWHAH) in meiotic spindle assembly during mouse oocyte maturation," (in eng), BMC Dev Biol, vol. 13, p. 10, Apr 12013.

[26] S. De and D. Kline, "Erratum to: evidence for the requirement of 14-3-3eta (YWHAH) in meiotic spindle assembly during mouse oocyte maturation," BMC developmental biology, vol. 14, no. 1, p. 20, 2014.

[27] S. De, J. Marcinkiewicz, and D. Kline, "Expression of 143-3 Protein Isoforms in Different Stages of Follicular Development in Adult Mouse Ovaries," (in English), Biology of Reproduction, vol. 85, Jul 2011.

[28] S. De, J. L. Marcinkiewicz, S. Vijayaraghavan, and D. Kline, "Expression of 14-3-3 protein isoforms in mouse oocytes, eggs and ovarian follicular development," BMC Res Notes, vol. 5, p. 57, Jan 232012.

[29] S. De, A. Reese, and D. Kline, "Interactions of 14-3-3 (YWHA) protein isoforms with CDC25B phosphatase in mouse oocytes," Mol Biol Cell, vol. 23, 2012.

[30] S. De, B. F. Villarreal, S. Vijayaraghavan, and D. Kline, "Identification of 14-3-3 Protein Isoforms and their Differential Subcellular Distribution in Mouse Oocytes and Eggs," Mol Biol Cell vol. 22, 2011.

[31] A. A. Eisa et al., "YWHA (14-3-3) protein isoforms and their interactions with $\mathrm{CDC} 25 \mathrm{~B}$ phosphatase in mouse oogenesis and oocyte maturation," BMC Developmental Biology, vol. 19, no. 1, p. 20, 2019/10/22 2019.

[32] A. Aitken, S. Howell, D. Jones, J. Madrazo, and Y. Patel, "14-3-3 alpha and delta are the phosphorylated forms of raf-activating 14-3-3 beta and zeta. In vivo stoichiometric phosphorylation in brain at a Ser-Pro-Glu-Lys MOTIF," $J$ Biol Chem, vol. 270, no. 11, pp. 5706-9, Mar 171995. 
[33] E. M. Skoulakis and R. L. Davis, "14-3-3 proteins in neuronal development and function," Mol Neurobiol, vol. 16, no. 3, pp. 269-84, Jun 1998.

[34] C. S. Karam, W. A. Kellner, N. Takenaka, A. W. Clemmons, and V. G. Corces, "14-3-3 mediates histone cross-talk during transcription elongation in Drosophila," PLoS Genet, vol. 6, no. 6, p. e1000975, Jun 32010.

[35] R. Benton, I. M. Palacios, and D. St Johnston, "Drosophila 14-3-3/PAR-5 is an essential mediator of PAR-1 function in axis formation," Dev Cell, vol. 3, no. 5, pp. 659-71, Nov 2002.

[36] R. Benton and D. St Johnston, "Drosophila PAR-1 and 143-3 inhibit Bazooka/PAR-3 to establish complementary cortical domains in polarized cells," Cell, vol. 115, no. 6 , pp. 691-704, Dec 122003.

[37] L. Kockel, G. Vorbruggen, H. Jackle, M. Mlodzik, and D. Bohmann, "Requirement for Drosophila 14-3-3 zeta in Raf-dependent photoreceptor development," Genes Dev, vol. 11, no. 9, pp. 1140-7, May 11997.

[38] N. Philip, S. F. Acevedo, and E. M. Skoulakis, "Conditional rescue of olfactory learning and memory defects in mutants of the 14-3-3 $\zeta$ gene leonardo," $J$ Neurosci., vol. 21, no. 21, pp. 8417-8425, 2001.

[39] H. C. Chang and G. M. Rubin, "14-3-3 epsilon positively regulates Ras-mediated signaling in Drosophila," Genes Dev, vol. 11, no. 9, pp. 1132-9, May 11997.

[40] A. C. Tien, H. Y. Hsei, and C. T. Chien, "Dynamic expression and cellular localization of the drosophila 143-3epsilon during embryonic development," Mech Dev, vol. 81, no. 1-2, pp. 209-12, Mar 1999.

[41] S. F. Acevedo, K. K. Tsigkari, S. Grammenoudi, and E. M. Skoulakis, "In vivo functional specificity and homeostasis of Drosophila 14-3-3 proteins," Genetics, vol. 177, no. 1, pp. 239-53, Sep 2007.

[42] K. K. Tsigkari, S. F. Acevedo, and E. M. Skoulakis, "14$3-3 \varepsilon$ is required for germ cell migration in Drosophila," PLoS One, vol. 7, no. 5, p. e36702, 2012.

[43] M. D. Nielsen, X. Luo, B. Biteau, K. Syverson, and H. Jasper, "14-3-3 Epsilon antagonizes FoxO to control growth, apoptosis and longevity in Drosophila," Aging Cell, vol. 7, no. 5, pp. 688-99, Oct 2008.

[44] T. Shandala et al., "14-3-3e has a crucial role in antimicrobial peptide secretion and innate immunity," J Cell Sci., vol. 124, no. 13, pp. 2165-2174, 2011.

[45] W. Li, E. M. Skoulakis, R. L. Davis, and N. Perrimon, "The Drosophila 14-3-3 protein Leonardo enhances Torso signaling through D-Raf in a Ras 1-dependent manner," Development, vol. 124, no. 20, pp. 4163-71, Oct 1997.

[46] K. Broadie, E. Rushton, E. M. Skoulakis, and R. L. Davis, "Leonardo, a Drosophila 14-3-3 protein involved in learning, regulates presynaptic function," Neuron, vol. 19, no. 2, pp. 391-402, Aug 1997.

[47] T. T. Su, D. H. Parry, B. Donahoe, C. T. Chien, P. H. O'Farrell, and A. Purdy, "Cell cycle roles for two 14-3-3 proteins during Drosophila development," J Cell Sci, vol. 114, no. Pt 19, pp. 3445-54, Oct 2001.

[48] Z. Huang, K. Myers, B. Khatra, and S. Vijayaraghavan, "Protein 14-3-3zeta binds to protein phosphatase PP1gamma2 in bovine epididymal spermatozoa," Biol Reprod, vol. 71, no. 1, pp. 177-84, Jul 2004.

[49] P. Puri, K. Myers, D. Kline, and S. Vijayaraghavan, "Proteomic analysis of bovine sperm YWHA binding partners identify proteins involved in signaling and metabolism," Biol Reprod, vol. 79, no. 6, pp. 1183-91, Dec 2008.
[50] P. Puri, A. Acker-Palmer, R. Stahler, Y. Chen, D. Kline, and S. Vijayaraghavan, "Identification of testis 14-3-3 binding proteins by tandem affinity purification," Spermatogenesis, vol. 1, no. 4, pp. 354-365, Oct 2011.

Publish your research article in AIJR journals-

$\checkmark \quad$ Online Submission and Tracking

$\checkmark$ Peer-Reviewed

$\checkmark$ Rapid decision

$\checkmark \quad$ Immediate Publication after acceptance

$\checkmark \quad$ Articles freely available online

$\checkmark \quad$ Retain full copyright of your article.

Submit your article at journals.aijr.in

Publish your books with AIJR publisher-

$\checkmark \quad$ Publish with ISBN and DOI.

$\checkmark$ Publish Thesis/Dissertation as Monograph.

$\checkmark$ Publish Book Monograph.

$\checkmark$ Publish Edited Volume/ Book.

$\checkmark \quad$ Publish Conference Proceedings

$\checkmark \quad$ Retain full copyright of your books.

Submit your manuscript at books.aijr.org 